\section{Anomalously small magnetic field in the local interstellar cloud}

\section{George Gloeckler ${ }^{\star} \dagger$, L. A. Fisk $\dagger$ \& Johannes Geiss $\neq$}

* Department of Physics, University of Maryland, College Park, Maryland 20742, USA

$\dagger$ Department of Atmospheric, Oceanic, and Space Sciences, University of Michigan, Ann Arbor, Michigan 48109, USA

$\ddagger$ International Space Science Institute, Hallerstrasse 6, CH-3012 Bern, Switzerland

The solar wind carves out a cavity, known as the heliosphere, in the warm local interstellar cloud, which is itself embedded in a larger hot cloud. It is generally assumed that there is an overall pressure balance between these three regions. Thermal pressure and magnetic field pressure in the local interstellar cloud should therefore balance the inward pressure from the hot cloud ${ }^{1-3}$, and determine the size of the heliosphere. Here we present direct measurements of the density and speed of interstellar hydrogen and helium ions deep inside the Solar System, from which we derive the thermal pressure in the local interstellar cloud. Combined with the fact that the magnetic field strength in the local cloud is constrained to be less than $4.3 \mu \mathrm{G}$ (to be compatible with the fact that the Voyager 1 spacecraft has yet to encounter the heliosphere termination shock), the total pressure that we infer is insufficient to balance the inward pressure from the hot cloud. We conclude that either the magnetic field in the local cloud is inhomogeneous, or there is a significant, as yet undetected, non-thermal component to the pressure in the local cloud.

Interstellar hydrogen and helium atoms enter the Solar System owing to its relative motion with respect to the local interstellar cloud. Influenced only by solar gravity, these neutral species penetrate deep into the heliosphere, where some are ionized by solar radiation. We detect these ions (called pickup ions ${ }^{4-7}$ ) with the Ulysses spacecraft in the inner heliosphere between 2.5 and $5 \mathrm{AU}$ from the Sun, and use these measurements to obtain densities of interstellar neutral and ionized hydrogen and helium in the very local interstellar cloud, that is, within a few thousand aU from the Sun.

The density of interstellar neutral hydrogen reaching the inner heliosphere is significantly reduced by ionization in two distinct locations. The first, called the filtration region, is in the local interstellar cloud (LIC) just beyond the heliopause, a boundary separating solar from interstellar plasma. In this region (roughly 100-200 $\mathrm{AU}$ wide $^{8-11}$ ) the LIC plasma, forced to move around the heliopause, slows down and in a process known as filtration ${ }^{8-10}$, converts some fraction of the $\mathrm{H}$ atoms to fast protons by protonhydrogen charge-exchange. The ram and thermal pressure of the newly created protons and the original LIC plasma, combined with the pressure of the LIC magnetic field draped around the heliopause, determine the size of the heliosphere. The amount of reduction of atomic hydrogen passing through the filtration region is roughly proportional to its average proton density.

The second location where ionization occurs is inside the heliosphere, where both interstellar atomic $\mathrm{H}$ and $\mathrm{He}$ are ionized by the solar wind and solar ultraviolet radiation; the ionization rate (which we measure directly) is strongest near the Sun. The newly ionized particles, called pickup ions, are accelerated and swept away from the Sun by the solar wind. It is possible to observe pickup $\mathrm{He}^{2+}$ and $\mathrm{H}^{+}$and distinguish them from the far more abundant solarwind $\mathrm{He}^{2+}$ and $\mathrm{H}^{+}$by their speed distribution as shown in Figs 1 and 2.
Figure 1 shows the speed distribution of $\mathrm{He}^{2+}$ measured with the Solar Wind Ion Composition Spectrometer ${ }^{12}$ (SWICS) on the Ulysses spacecraft. Pickup $\mathrm{He}^{2+}$ is produced predominantly by double charge-exchange with solar-wind $\alpha$-particles and its rate of production from neutral He is known from SWICS measurements of the solar-wind $\mathrm{He}^{2+}$ flux. The best fit to the observed pickup $\mathrm{He}^{2+}$ spectrum both above and below the solar-wind peak yields an interstellar neutral helium density, $n\left(\mathrm{He}_{\mathrm{TS}}\right)=$ $0.0153 \pm 0.0018 \mathrm{~cm}^{-3}$. Our result is in excellent agreement with the value of $0.0155 \pm 0.0015 \mathrm{~cm}^{-3}$ derived from direct measurements of interstellar neutral helium on Ulysses ${ }^{13}$. Because $\mathrm{He}$ is not affected by filtration, $n\left(\mathrm{He}_{\mathrm{I}}\right)$, the neutral-helium density in the LIC, is equal to $n\left(\mathrm{He}_{\mathrm{TS}}\right)$.

The distribution function of protons measured near the ecliptic plane at distances $\sim 4.5$ AU from the Sun is shown in Fig. 2. Using the 'hot' model of Thomas ${ }^{14}$ with an interstellar gas temperature of $7,500 \mathrm{~K}$, we calculate the expected distribution of pickup $\mathrm{H}^{+}$(solid curve). The shape of the distribution fixes the only free parameter of the model $^{6,7}, \beta_{\text {Hloss }}$, the rate at which hydrogen atoms are ionized at $1 \mathrm{AU}$ in the heliosphere. The phase space density between 1.5 and twice the solar wind speed $(1.5<W<2)$ gives the product of the density of interstellar neutral hydrogen in the outer heliosphere, $n\left(\mathrm{H}_{\mathrm{TS}}\right)$, and the rate of production of pickup $\mathrm{H}^{+}$. From the best fit to the observed pickup $\mathrm{H}^{+}$distribution we find $n\left(\mathrm{H}_{\mathrm{TS}}\right)=$ $0.115 \pm 0.025 \mathrm{~cm}^{-3}$. Previously, $n\left(\mathrm{H}_{\mathrm{TS}}\right)$ has been deduced from observations of resonant back-scattering of solar ultraviolet radiation $^{3,15}$, but these methods depend on complex back-scattering models with poorly known parameters, and often have systematic errors due to calibration uncertainties ${ }^{16}$. Neutral-hydrogen densities based on ultraviolet observations, revised for more realistic $\beta_{\text {Hloss }}$ rates $^{15}$, are widely scattered from 0.03 to $0.3 \mathrm{~cm}^{-3}$ with an average value of $\sim 0.135 \mathrm{~cm}^{-3}$, which agrees with our result.

Combining our values for the densities of atomic $\mathrm{H}$ and $\mathrm{He}$ at the termination shock with measured parameters in the LIC listed in Table 1 , we evaluate the atomic $\mathrm{H}$ density in the LIC, $n\left(\mathrm{H}_{\mathrm{I}}\right)$, and the average proton density in the filtration region, $\langle n(\mathrm{p})\rangle$. The computational steps and results are given in Table 1 . We find that $n\left(\mathrm{H}_{\mathrm{I}}\right)=0.20 \pm 0.03 \mathrm{~cm}^{-3}$, and that $\sim 40 \%$ of the LIC atomic hydrogen is excluded from the heliosphere by charge exchange in the filtration region.

A LIC magnetic field of $B<3.7 \mu \mathrm{G}$, when taken with values ( T, R2 and $\left.n\left(\mathrm{H}_{\mathrm{II}}\right)=\langle n(\mathrm{p})\rangle\right)$ from Table 1 , shows that the Sun moves supersonically relative to the LIC. Assuming $B<3.7 \mu \mathrm{G}$, there will be a bow shock at the outer boundary of the filtration region. The magnetic field orientation observed to be nearly paralle ${ }^{17}$ to the shock surface will make it a perpendicular MHD shock. Using standard jump conditions ${ }^{18}$, and parameters from Table 1 , we find the total LIC pressure, $P_{\text {LIC }}(r)$, at the heliopause as a function of the shock compression ratio, $r$, we use the magnetic field and densities of atomic hydrogen, $n(\mathrm{H} \mathrm{I})$, protons, $n(\mathrm{H} \mathrm{II})$, and ionized helium, $n$ (He II) in the LIC just beyond the bow shock. The location, $R_{\mathrm{TS}}$, of the termination shock (a boundary where the supersonic solar wind becomes subsonic) is found from pressure balance between the solar wind and the LIC plasmas at the heliopause. The solar-wind pressure at the heliopause, $P_{\mathrm{SW}}\left(R_{\mathrm{TS}}\right)$, depends on the solar-wind density and speed just inside the termination shock and decreases rapidly with $R_{\mathrm{TS}}$. Thus all LIC parameters, such as $B$, calculated as a function of the compression ratio may also be expressed in terms of $R_{\mathrm{TS}}$. (See Fig. 3 legend for computational details.)

In Fig. 3a we show the dependence of the computed magnetic field on the location of the heliospheric termination shock. The shaded vertical region indicates the range of $R_{\mathrm{TS}}$ values derived from observations ${ }^{19}$ of anomalous cosmic rays with Voyagers 1 and 2; the minimum distance to the termination shock is at $80 \mathrm{AU}$. This places an upper limit on $B$ of $3 \mu \mathrm{G}$ (upper dashed curve in Fig. 3a), justifying our assumption of supersonic flow. Our most probable calculated field (solid curve) agrees remarkably well with reported 


\section{Table 1 Measured and derived average parameters in the local interstellar cloud}

\begin{tabular}{|c|c|c|c|}
\hline Parameter & Units & $\begin{array}{l}\text { Local* interstellar } \\
\text { cloud }\end{array}$ & $\begin{array}{l}\text { Very local interstellar } \\
\text { cloud }(\leqslant 2,000 \mathrm{AU})\end{array}$ \\
\hline$n\left(\mathrm{H}_{T S}\right)$ & $\mathrm{cm}^{-3}$ & & $0.115 \pm 0.025 t$ \\
\hline$n\left(\mathrm{He}_{1}\right)=n\left(\mathrm{He}_{\mathrm{TS}}\right)$ & $\mathrm{cm}^{-3}$ & & $0.0153 \pm 0.0018 \dagger$ \\
\hline$\dddot{V_{0}}$ & $\mathrm{kms}^{-1}$ & & $25.3 \pm 0.4 \ddagger$ \\
\hline$\dddot{T}$ & K & $7,000 \pm 600 \ddagger$ & \\
\hline $\mathrm{R} 1=n\left(\mathrm{H}_{1}\right) / n\left(\mathrm{He}_{1}\right)$ & & $13 \pm 1 \S$ & \\
\hline $\mathrm{R} 2=n(\mathrm{H}) / n(\mathrm{He})$ & & $10 \|$ & \\
\hline$n\left(\mathrm{H}_{1}\right)=\mathrm{R} 1 \times n\left(\mathrm{He}_{1}\right)$ & $\mathrm{cm}^{-3}$ & & $0.20 \pm 0.03$ \\
\hline$\widetilde{F}=n\left(\mathrm{H}_{\mathrm{TS}}\right) / n\left(\mathrm{H}_{1}\right)=$ filtration factor & & & $0.58 \pm 0.15$ \\
\hline$\langle n(p)\rangle=(-1 / L \sigma) \mid \ln (F / 1.46)$ & $\mathrm{cm}^{-3}$ & & $0.073 \pm 0.0315$ \\
\hline$n(\mathrm{H} \|)=n(\mathrm{p}) / r$ & $\mathrm{~cm}^{-3}$ & & $0.040 \pm 0.017 \#$ \\
\hline$n\left(H_{)}=n\left(H_{1}\right)+n\left(H_{11}\right)\right.$ & $\mathrm{cm}^{-3}$ & & $0.240 \pm 0.034 \#$ \\
\hline$n\left(\mathrm{He}_{\|}\right)=[n(\mathrm{H})] / \mathrm{R}-n\left(\mathrm{He}_{1}\right)$ & $\mathrm{cm}^{-3}$ & & $0.009 \pm 0.004 \#$ \\
\hline$n_{e}=n\left(\mathrm{H}_{\|}\right)+n\left(\mathrm{He}_{\|}\right)$ & $\mathrm{cm}^{-3}$ & & $0.049 \pm 0.016$ \\
\hline$X_{H}=n\left(H_{11}\right) / n(H)$ & & $0.17 \nrightarrow<X_{H}<0.4 \$$ & $0.18 \pm 0.01 \#^{* *}$ \\
\hline$X_{\mathrm{He}}=n\left(\mathrm{He}_{\|}\right)(\mathrm{R} 2 / n(\mathrm{H}))$ & & $0.3 \leadsto<X_{\mathrm{He}}<0.5 \$$ & $0.36 \pm 0.02 \# * *$ \\
\hline
\end{tabular}

*Average values measured or calculated over distances of a few to $\sim 100 \mathrm{pc}$ and assumed to be also valid in the immediate neighbourhood of the heliosphere.

$\dagger$ Densities near the termination shock, present work.

$\ddagger$ From refs 13,24 .

$\$$ From ref. 21.

II From ref. 25.

- Proton density in the filtration region: the width of the filtration region is $L=160 \pm 40 \mathrm{Au}$ (refs 8 - 11 ), and $\sigma=5.3 \times 10^{-15} \mathrm{~cm}^{2}$ is the proton-hydrogen charge-exchange cross-section ${ }^{26}$ at $V_{\text {rel }}=19 \mathrm{~km} \mathrm{~s}^{-1}$, the average relative speed between protons and $\mathrm{H}$ atoms in the filtration region. The factor of $1.46=V_{\text {rel }} /\left(V_{\text {rel }}-6\right)$ accounts for that fraction of slow-moving $\mathrm{H}$ atoms, produced by charge exchange, that enter the heliosphere, contribute to $n\left(\mathrm{H}_{T S}\right)$, and reduce the average speed of interstellar $\mathrm{H}$ inside the heliosphere ${ }^{27}$ to $20 \mathrm{~km} \mathrm{~s}^{-}$

\# For compression ratio, $r=1.8$

in From ref. 22.

** For $80<R_{\text {TS }}<85 \mathrm{AU}$ see Fig. 3 legend for error limits.

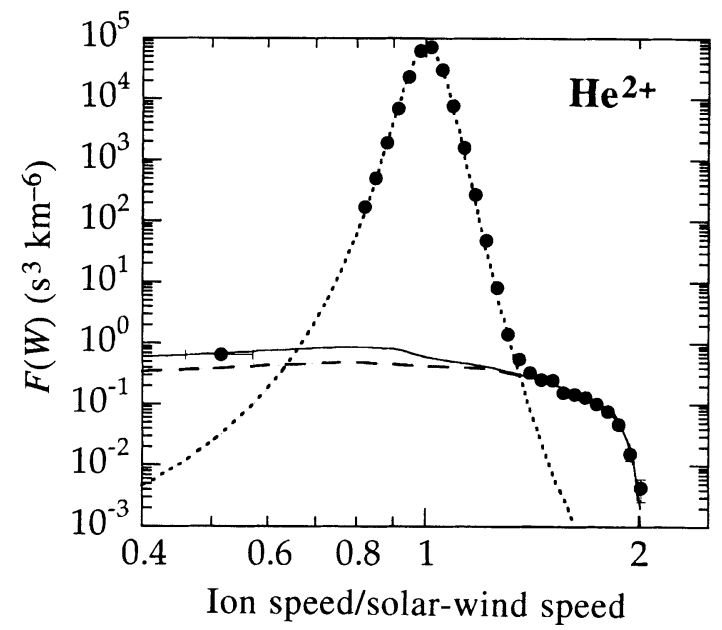

Figure 1 Phase space density of $\mathrm{He}^{2+}$ versus $W$, the ion speed in the spacecraft frame of reference divided by the solar-wind speed. This time-averaged spectrum was observed with SWICS for 111 days (19 July to 8 October, 1994) at $\sim 2.5 \mathrm{AU}$ and $\sim 70^{\circ}$ latitude in the persistently quiet high-speed $\left(\sim 808 \mathrm{~km} \mathrm{~s}^{-1}\right)$ solar wind. Long observation periods and quiet solar-wind conditions were required to observe these rare pickup ions. The dotted curve is the fit to the solar-wind $\mathrm{He}^{2+}$, yielding a density of $0.022 \mathrm{~cm}^{-3}$ and a thermal speed of $29 \mathrm{~km} \mathrm{~s}^{-1}$. The dashed curve is computed assuming that the pickup ion distribution function is isotropic. For the heliospheric spatial distribution of interstellar atomic He we use the 'hot' model ${ }^{14}$ with $\mu$ (radiation pressure divided by the gravitation force) of 0 , a loss rate ${ }^{7}$ of $0.6 \times 10^{-7} \mathrm{~s}^{-1}$, and He with speed of $26 \mathrm{~km} \mathrm{~s}^{-1}$ and temperature of $7,500 \mathrm{~K}$. The production rate is measured to be $2.0 \times 10^{-9} \mathrm{~s}^{-1}$, being almost entirely due to double charge-exchange with solar-wind $\alpha$-particles $\left(\sigma=1.8 \times 10^{-16} \mathrm{~cm}^{2}\right)^{28}$. Because at high latitudes the average magnetic field is nearly radial, anisotropic pickup ion distributions are expected ${ }^{29,30}$. The best fit (solid curve) for $1.7<W<2$ is obtained with $n\left(\mathrm{HE}_{\mathrm{TS}}\right)=0.0153 \pm 0.018 \mathrm{~cm}^{-3}$ using a $25 \%$ anisotropy correction. We did not use here the far more abundant $\mathrm{He}^{+}$, discovered by Möbius ${ }^{4}$ and also observed with SWICS, to find $n\left(\mathrm{He}_{\mathrm{TS}}\right)$ because during the time period of our observations its production rate from photoionization was not known owing to lack of direct measurements. Details of how the observed and model distributions are computed are given in refs 6 and 7 .

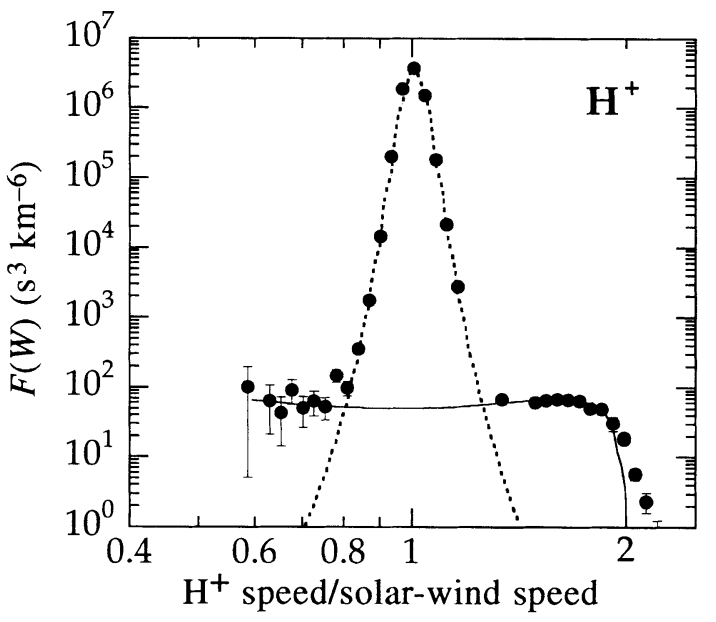

Figure 2 As Fig. 1 for $\mathrm{H}^{+}$. This spectrum, $F(W)$, was measured during a 16-day quiet period in late 1991 when Ulysses was at $\sim 4.88 \mathrm{Au}$ in the ecliptic plane. In this period of relatively cool solar wind, the pickup $\mathrm{H}^{+}$is well separated from the far more abundant solar-wind protons centred at $W=1$. The dotted curve is the fit to the solar-wind distribution using a convected maxwellian distribution with density of $0.09 \mathrm{~cm}^{-3}$ and thermal speed of $10.5 \mathrm{~km} \mathrm{~s}^{-1}$. As the average heliospheric magnetic field direction was nearly perpendicular to the solar-wind flow direction, the pickup ion distribution was essentially isotropic, and no anisotropy corrections $\mathrm{s}^{29,30}$ were required in determining interstellar densities. The solid curve is the fit to the pickup ion spectrum. We use the 'hot' mode $\left.\right|^{14}$ to compute the heliospheric spatial distribution of neutral interstellar hydrogen with a speed ${ }^{27}$ of $20 \mathrm{~km} \mathrm{~s}^{-1}$ and temperature of 7,500 K; $\mu$ is taken as 1 , and the isotropic form of the pickup ion speed distribution in the solar-wind frame ${ }^{30}$ is used. With $\mu=1$ the shape of the observed $F(W)$ for $1.25<W<1.9$ restricts $\beta_{\text {Hloss, }}$, the loss rate of atomic $\mathrm{H}$, to $(8.5 \pm 1) \times 10^{-7} \mathrm{~S}^{-1}$. The best fit is obtained with $n\left(\mathrm{H}_{\mathrm{TS}}\right)=0.115 \pm 0.025 \mathrm{~cm}^{-3}$ using $\beta_{\mathrm{Hprod}}=2.7 \times 10^{-7} \mathrm{~S}^{-1}$. The charge-exchange portion of $\beta_{\text {Hprod }}$ equals $1.7 \times 10^{-7} \mathrm{~s}^{-1}$, and is the product of the observed solarwind flux (adjusted to $1 \mathrm{AU}$ ) and the proton-hydrogen charge-exchange crosssection $^{26}, \quad \sigma_{0}=1.8 \times 10^{-15} \mathrm{~cm}^{2}$ at the solar-wind speed of $475 \mathrm{kms}^{-1}$ Photoionization ${ }^{31}$ accounts for the rest. 
values $^{17,20}$ of the interstellar magnetic field of $1.4 \pm 0.15 \mu \mathrm{G}$ represented by the shaded horizontal region.

The variation with $R_{\mathrm{TS}}$ of the fractional ionization of hydrogen, $X(\mathrm{H})$, and helium, $X(\mathrm{He})$, and the electron density, $n_{\mathrm{e}}$, in the very local interstellar cloud is shown in Fig. 3b. Our values for both $X(\mathrm{H})$ and $X(\mathrm{He})$ are well below measured ${ }^{21}$ upper limits given in Table 1 . However, imposing the calculated lower limit ${ }^{22}$ of 0.17 on $X(\mathrm{H})$ places an upper limit on $R_{\mathrm{TS}}$ of $\sim 85 \mathrm{AU}$. Thus, the most probable value of $B$ is between $\sim 1.3$ and $\sim 2 \mu \mathrm{G}$ for the case of supersonic

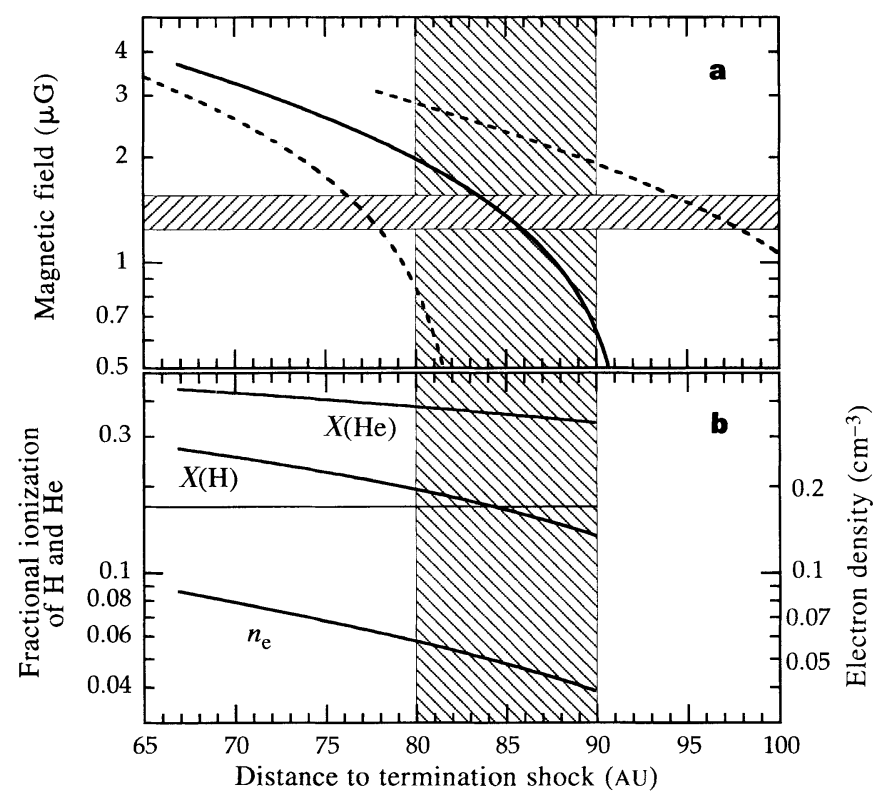

Figure $\mathbf{3}$ a, Variation of the calculated magnetic field strength, $B$, just beyond the bow shock with distance to the termination shock, $R_{\mathrm{TS}}$. Mean (solid curve) and $1 \sigma$ limits (dotted curves) are shown. $B^{2} / 8 \pi=\rho V_{0}^{2}\left[(4 / r)-1-5 r\left(2 n_{e} k T\right)\right] /(r+5)$, where $V_{0}=26 \mathrm{~km} \mathrm{~s}^{-1}$ is the relative speed of the Sun through the local cloud, $r$ is the shock compression ratio, $\rho=m_{H}\left[n\left(\mathrm{H}_{\|}\right)+4 n\left(\mathrm{He}_{\|}\right)\right], n(\mathrm{H} \|)=\langle n(\mathrm{p})\rangle / r$ and $n\left(\mathrm{He}_{\|}\right)=\left[n\left(\mathrm{H}_{1}\right)+n\left(\mathrm{H}_{\|}\right)\right] / \mathrm{R} 2-n\left(\mathrm{He}_{1}\right)$. The resulting stagnation pressure at the heliopause from the interstellar medium is then $P_{\text {LIC }}=\left[(7 / 8)\left(\rho V_{0}^{2}\right)\right]+$ $\left[(3 / 8)\left(2 n_{\mathrm{e}} k T\right)\right]+3\left(B^{2} / 8 \pi\right)+P_{\mathrm{H}}$, where $P_{\mathrm{H}}=f n\left(\mathrm{H}_{1}\right)\left[m_{\mathrm{H}} V_{0}^{2}+k T\right]$ is the pressure from that fraction $f=[1-\exp (-\langle n(p)\rangle L \sigma)]$ of hydrogen atoms converted to $26 \mathrm{~km} \mathrm{~s}^{-1}$ protons in the filtration region by charge exchange. Magnetic field pressure and $P_{H}$ contribute significantly to $P_{\text {LIC. }}$. We neglect contributions from cosmic rays (as the observed gradients and thus the resulting force should be small) and from dust. The stagnation pressure at the heliopause from the solar wind, $P_{\mathrm{SW}}$ is determined using the usual gas dynamic calculation, relating conditions just inside the termination shock, to those measured ${ }^{23}$ at 49.1 au by Voyager 2 . We assume that in the distant heliosphere the solar wind expands uniformly but is decelerated slightly in the process of picking up interstellar atoms, neglect magnetic field pressure but include thermal pressure from pickup ions ${ }^{23}$. $P_{\mathrm{SW}}=(7 / 8) m_{\mathrm{H}}\left[1.16 n_{\mathrm{SW}}(\mathrm{p})\right]\left[49.1 U_{\mathrm{SW}} / R_{\mathrm{TS}}\right]^{2}+(3 / 8)\left(m_{\mathrm{H}} / 7\right)\left(U_{\mathrm{SW}} / R_{\mathrm{TS}}\right) \beta_{\mathrm{prod}} n\left(\mathrm{H}_{\mathrm{TS}}\right)$, where $U_{\mathrm{SW}}=V_{\mathrm{SW}}\left[1-(6 / 7) \sigma_{\mathrm{p}} n\left(\mathrm{H}_{\mathrm{TS}}\right) R_{\mathrm{TS}}\right] /\left[1-(6 / 7) \sigma_{\mathrm{p}} n\left(\mathrm{H}_{\mathrm{TS}}\right) 49.1\right]$ is the reduced solar-wind speed due to momentum loss to pickup hydrogen, $n_{\mathrm{sw}}(p)=0.0017 \mathrm{~cm}^{-3}$, $V_{\mathrm{SW}}=534 \mathrm{~km} \mathrm{~s}^{-1}$ is the 1996 averaged Voyager 2 solar-wind proton density and speed at $49.1 \mathrm{AU}$ (ref. 23), the factor of 1.16 accounts for the solar-wind $\mathrm{He} / \mathrm{H}$ of $4 \%$, and $\beta_{\text {prod }}=7 \times 10^{-7} \mathrm{~s}^{-1}$. Assuming that there is no flow across or along the heliopause in the direction of motion of the Sun, the stagnation pressure on either side of the heliopause must be equal ${ }^{8}$. Parameter values from Table 1 are used to obtain solutions of the magnetic field as a function of $R_{\mathrm{TS}} . \mathbf{b}$, Average values of the electron density and ionization fractions of $\mathrm{H}$ and $\mathrm{He}$ calculated as a function of $R_{\text {TS }}$ using values from Table 1 and computational steps given above. The shaded vertical region indicates the range of locations of the termination shock determined by Stone et al. ${ }^{19}$. The horizontal line is the calculated lower limit ${ }^{22}$ of $X_{H}$. flow. The corresponding bow shock compression ratios range from 1.5 to 1.9 .

The LIC is embedded in a region of tenuous, hot gas $\sim 80 \mathrm{pc}$ across $^{1-3,17,20}$ with thermal pressure ${ }^{1}$ between $\sim 1.2 \times 10^{-12}$ and $2.7 \times 10^{-12} \mathrm{dyn} \mathrm{cm}^{-2}$. The LIC thermal pressure $\left(2 n_{\mathrm{e}} k T\right)$ is at most $0.2 \times 10^{-12} \mathrm{dyn}^{-2}$, thus contradicting the basic assumption of thermal pressure balance in the interstellar medium ${ }^{1,2}$. It has been suggested ${ }^{1,2}$ that pressure balance could be restored by adding sufficient LIC magnetic pressure. But this requires a minimum $B$ of $5-7 \mu \mathrm{G}$, which is clearly inconsistent with our upper limit of $3 \mu \mathrm{G}$.

Could we substantially increase our upper limit on $B$ of $3 \mu \mathrm{G}$ by assuming subsonic conditions with no bow shock? For subsonic flow the pressure from the interstellar medium at the heliopause becomes $^{8} \quad P_{\text {LIC }}=0.5 \rho V_{0}^{2}+2 n_{\mathrm{e}} k T+3 B^{2} / 8 \pi+P_{\mathrm{H}} \quad$ (see Fig. 3a legend for definition of symbols). Using values in Table 1 with a compression ratio of 1 (no bow shock) we find that $B>4.3 \mu \mathrm{G}$ will push the average location of the termination shock below the minimum of $80 \mathrm{AU}$ (ref. 19) to $70 \mathrm{AU}$. At that distance Voyager 1, now at $66 \mathrm{AU}$, would have occasionally intercepted the termination shock because of its $\pm(3-5)$ AU excursions from its average position (in response to the $\pm(10-15) \%$ changes in the solar-wind ram pressure typically observed ${ }^{23}$ over several months).

We now consider how to reconcile our extreme upper limit of $B=4.3 \mu \mathrm{G}$ with the minimum field of $5 \mu \mathrm{G}$ required to achieve pressure balance with the hot cloud. One can abandon the idea that the magnetic field is uniform throughout the local cloud. Our results apply only to a very limited region of the LIC, within a few thousand AU of the Sun. The local cloud is likely to be complex and inhomogeneous ${ }^{3}$. Thus it is not unreasonable to imagine that the LIC field near the Sun is weak $(\sim 1-2 \mu \mathrm{G})$ and becomes much stronger $(\sim 5-7 \mu \mathrm{G})$ near its interface with the hot cloud. On the other hand, if it is assumed that the field is uniform but small $(\sim 1 \mu \mathrm{G})$ it is still just possible to achieve pressure balance with the hot cloud and have the termination shock at $80 \mathrm{Au}$ by postulating the existence of substantial $\left(\sim 10^{-12} \mathrm{dyn}_{\mathrm{cm}}^{-2}\right)$ non-thermal pressure, perhaps residing in an undetected high-energy tail of the LIC plasma. The expected detection of the termination shock by Voyager 1 (within perhaps the next 5 years) should allow us to derive more accurate values for the magnetic field in the very local interstellar cloud.

Received 9 September 1996; accepted 4 February 1997.

1. Bowyer, S., Lieu, R., Sidher, S. D., Lampton, M. \& Kunde, J. Nature 375, 212-214 (1995).

2. Cox, D. Nature 375, 185-186 (1995).

3. Cox, D. P. \& Reynolds, R. J. Annu. Rev. Astron. Astrophys. 25, 303-344 (1987).

4. Möbìus, E. et al. Nature 318, 426-429 (1985).

5. Gloeckler, G. et al. Science 261, 70-73 (1993)

6. Gloeckler, G. \& Geiss, J. Nature 381, 210-212 (1996)

. Gloeckler, G. in Proc. 1st ISSI Workshop (eds von Steiger, R., Lallement, R. \& Lee, M. A.) Space Sci. Rev 78, 335-346 (1996)

8. Holzer, T. E. Annu. Rev. Astron. Astrophys. 27, 199-234 (1989).

9. Baranov, V. B. \& Malama, Y. G. J. Geophys. Res. 100, 755-761 (1995)

10. Williams, L. L., Hall, D. T., Pauls, H. L. \& Zank, G. P. Astrophys. J. (submitted).

11. Steinolfson, R. S. \& Gurnett, D. A. Geophys. Res. Lett. 22, 651-654 (1995).

12. Gloeckler, G. et al. Astron. Astrophys. Suppl. Ser. 92, 267-289 (1992).

13. Witte, M., Rosenbauer, H., Banaskiewicz, M. \& Fahr, H. J. Adv. Space Res. 13, 121-126 (1993).

14. Thomas, G. E. Annu. Rev. Earth Planet. Sci. 6, 173-204 (1978).

15. Quemerais, E., Bertaux, J.-L., Sandel, B. R. \& Lallement, R. Astron. Astrophys. 290, 941-954 (1996).

16. Vallerga, J. in Proc. 1st ISSI Workshop (eds von Steiger, R., Lallement, R. \& Lee, M. A.) Space Sci. Rev. 78, 277-288 (1996)

17. Frisch, P. C. Space Sci. Rev. 72, 499-592 (1995).

18. Siscoe, G. L. in Solar System Magnetohydrodynamics: Concepts and Basic Equations in Solar Terrestria Physics (eds Carovillano, R. L. \& Forbes, J. M.) 74-76 (Reidel, Dordrecht, 1983).

19. Stone, E. C. Cummings, A. C. \& Webber, W. R. J. Geophys. Res. 101, 11017-11025 (1996).

20. Frisch, P. C. in Cosmic Winds and the Heliosphere (eds Jokipii, J. R., Sonett, C. P. \& Giampapa, M. S.) (Univ. Arizona Press, Tucson, in the press).

21. Dupuis, J., Vennes, S., Bowyer, S., Pradhan, A. K. \& Thejll, P. Astrophys. J. 455, 574-589 (1995). 22. Cheng, K.-P. \& Bruhweiler, F. C. Astrophys. J. 364, 573-581 (1990).

23. Burlaga, L. F., Ness, N. F., Belcher, I. W., Lazarus, A. I. \& Richardson, J. D. in Proc. Ist ISSI Workshop (eds von Steiger, R., Lallement, R. \& Lee, M. A.) Space Sci. Rev. 78, 33-42 (1996).

24. Geiss, J. \& Witte, M. in Proc. Ist ISSI Workshop (eds von Steiger, R., Lallement, R. \& Lee, M. A.) Space Sci. Rev. 78, 229-238 (1996).

25. Anders, E. \& Grevesse, N. Geochim. Cosmochim. Acta. 53, 197-214 (1988).

26. Fite, W. L., Smith, A. C. H. \& Stebbings, R. F. Proc. R. Soc. Lond. A 268, 527-534 (1962).

27. Lallement, R., Bertaux, J.-L. \& Clarke, J. T. Science 260, 1095-1098 (1993).

28. Janev, R. K., Langer, W. D., Evans, K. Jr \& Post, D. E. Elementary Processes in Hydrogen-Helium Plasmas (Cross Sections and Reaction Rate Coefficients) (Springer, Berlin, 1987). 
29. Gloeckler, G., Schwadron, N. A., Fisk, L. A. \& Geiss, J. Geophys. Res. Lett. 22, 2665-2668 (1995). 30. Fisk, L. A., Schwadron, N. A. \& Gloeckler, G. Geophys. Res. Lett. 24, $93-96$ (1997).

31. Rucinski, D. et al. Proc. 1st ISSI Workshop (eds von Steiger, R., Lallement, R. \& Lee, M. A.) Space Sci. Rev. 78, 73-84 (1996).

Acknowledgements. The SWICS instrument was developed by a collaboration of the universities of Maryland, Bern and Braunschweig, and the Max-Planck-Institut für Aeronomie. We thank C. Gloeckler for her help with data reduction, and P. C. Frisch, E. Möbius and D. A. Gurnett for discussions. This work was supported by NASA/JPL and the Swiss National Science Foundation.

Correspondence should be addressed to G.G. (e-mail: gloeckler@umdsp.umd.edu).

\section{Storage of hydrogen in} single-walled carbon nanotubes

A. C. Dillon*, K. M. Jones*, T. A. Bekkedahl*, C. H. Kiang $\dagger$, D. S. Bethune $\nmid$ \& M. J. Heben ${ }^{*}$

* National Renewable Energy Laboratory, 1617 Cole Boulevard, Golden, Colorado 80401-3393, USA

† IBM Research Division, Almaden Research Center, 650 Harry Road, San Jose, California 95120-6099, USA

Pores of molecular dimensions can adsorb large quantities of gases owing to the enhanced density of the adsorbed material inside the pores ${ }^{1}$, a consequence of the attractive potential of the pore walls. Pederson and Broughton have suggested ${ }^{2}$ that carbon nanotubes, which have diameters of typically a few nanometres, should be able to draw up liquids by capillarity, and this effect has been seen for low-surface-tension liquids in large-diameter, multi-walled nanotubes ${ }^{3}$. Here we show that a gas can condense to high density inside narrow, single-walled nanotubes (SWNTs). Temperature-programmed desorption spectrosocopy shows that hydrogen will condense inside SWNTs under conditions that do not induce adsorption within a standard mesoporous activated carbon. The very high hydrogen uptake in these materials suggests that they might be effective as a hydrogen-storage material for fuel-cell electric vehicles.

Soots containing SWNTs were synthesized by co-evaporation of cobalt and graphite in an electric arc ${ }^{4}$. Amorphous carbon-coated fibres several micrometres in length were observed in the soots by transmission electron microscopy (TEM). Fibres typically consisted of 7-14 bundled SWNTs which were individually $\sim 12 \AA$ in diameter ${ }^{5}$. Cobalt nanoparticles, $5-50 \mathrm{~nm}$ in diameter, were also present, embedded in amorphous carbon. Electron microprobe analysis measured cobalt contents of $\sim 20 \mathrm{wt} \%$. The remainder of each sample consisted of amorphous carbon and planar graphitic fractions. Activated carbon (AC) was prepared from pitch precursors, activated by $\mathrm{KOH}$, and exhibited a narrow peak in the porevolume distribution of $30 \AA$ with a full-width at half-maximum of $\sim 20 \AA$. (Material and pore analysis was supplied by Spectracorp Ltd.) Extensive TEM examinations showed the AC to be similar in structure and overall morphology to the arc-generated soots except for the absence of SWNTs and cobalt nanoparticles.

The adsorption of $\mathrm{H}_{2}$ on SWNT soots and AC was probed with temperature programmed desorption (TPD) spectroscopy in an ultra-high-vacuum chamber equipped with a liquid-nitrogencooled cryostat and a mass spectrometer. Samples weighing $\sim 1 \mathrm{mg}$ were contained in a platinum foil packet with pinholes for gas diffusion. The packet temperature was measured with a thermocouple and controlled by resistive heating. TPD experiments were performed at $1 \mathrm{~K} \mathrm{~s}^{-1}$, and standard $\mathrm{H}_{2}$ exposures were carried out at 300 torr for 10 minutes at $273 \mathrm{~K}$ followed by 3 minutes at $133 \mathrm{~K}$ except where noted. The samples were cooled to $90 \mathrm{~K}$ while the chamber was evacuated to $<5 \times 10^{-8}$ torr before TPD.

$\mathrm{H}_{2}$ desorbs from as-prepared SWNT soots (Fig. 1a) and AC (Fig. 1b) within the same temperature range, but with differing intensities. The signals are peaked just above $133 \mathrm{~K}$ because the $\mathrm{H}_{2}$ pressure was reduced as the sample was cooled from $133 \mathrm{~K}$ to $90 \mathrm{~K}$. Planar graphite cannot stabilize $\mathrm{H}_{2}$ under these exposure conditions ${ }^{5}$, so desorption must be from nanoporous environments ${ }^{6}$ associated with the high-surface-area amorphous carbon fractions present in both materials. The signal from the SWNT samples (Fig. 1a) is $\sim 10$ times as great as the signal from AC (Fig. 1b), consistent with the higher amorphous carbon content (as
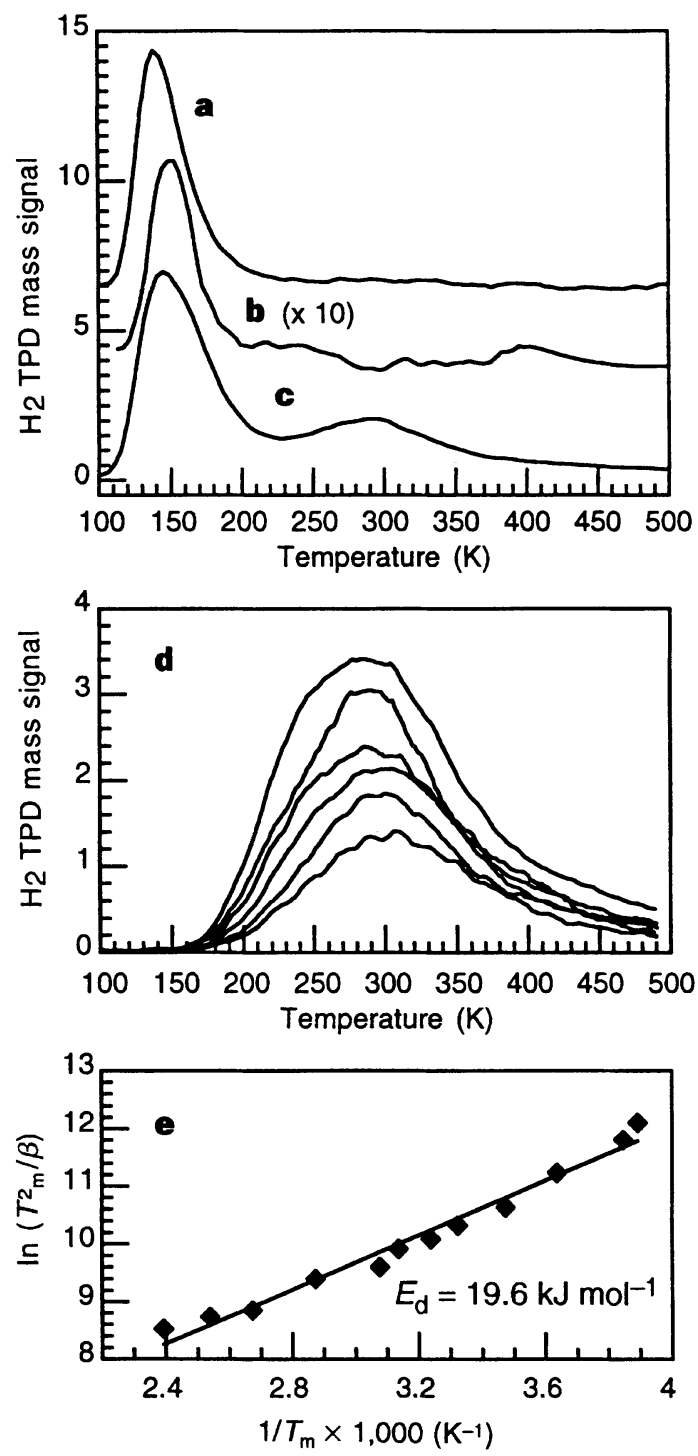

Figure 1 Temperature programmed desorption data. a, TPD spectrum from asproduced SWNT sample after standard hydrogen exposure. b. TPD spectrum from activated carbon sample, magnified 10 times, after standard hydrogen exposure. c. TPD spectrum from SWNT sample after heating in vacuum to $970 \mathrm{~K}$ and standard hydrogen exposure. d. Hydrogen desorption signals after exposures that populated only the high-temperature sites. Coverages range from 0.3 to saturation. e. Plot of $\ln \left(T_{\mathrm{m}}^{2} / \beta\right)$ against $1 / T_{\mathrm{m}}$ with $\beta$ varying from 0.3 to $30 \mathrm{Ks}^{-1}$. The activation energy for desorption, $E_{\mathrm{d}}$, is the slope of the line $\left(19.6 \mathrm{~kJ} \mathrm{~mol}^{-1}\right)$. 